\title{
Study of multi physical parameter monitoring device based on FBG sensors demodulation system
}

\author{
Qi Li, Li-Hui Feng and Ai-Ying Yang \\ School of Optoelectronics, Beijing Institute of Technology, Beijing, China \\ E-mail: lihui.feng@bit.edu.cn \\ http://www.bit.edu.cn
}

\begin{abstract}
For cross-sensitivity problem between strain and temperature in Fiber Bragg Grating (FBG), A FBG signal demodulation system is designed in this paper; signals are acquired and processed by Labview. The experiment of strain sensing with real-time temperature compensation has been studied based on FBG signal demodulation system. The results show that FBG center wavelength drift has good linear relationship with strain. Wavelength resolution of the system is $1 \mathrm{pm}$; detected wavelength range is $54 \mathrm{~nm}$. Range of sensing temperature demodulation is $-10^{\circ} \mathrm{C}-70^{\circ} \mathrm{C}$. The range of sensing strain demodulation is more than $100 \mu \varepsilon$. When temperature increases from $25^{\circ} \mathrm{C}$ to $50^{\circ} \mathrm{C}$, TC FBG can provide accurate temperature compensation for strain FBG in strain measurement. This system can be used as multi physical parameter monitoring device of the airplane status.
\end{abstract}

Keywords: FBG; Strain Sensor; Temperature Sensor; Wavelength Drift.

\section{Introduction}

FBG sensor is a light passive component, it is widely used in the fields of optical communications and sensing. FBG has the following great characteristics: high stability, great repeatability, dielectric, anti-electric magnetic field interference, anti-corrosion, good spectral characteristics[1,2]. So FBG has huge tremendous value for smart structure monitoring, intelligent oil wells and pipelines, intelligent civil engineering construction, aerospace and voyage engineering[3]. In the field of the airplane Status monitoring, many physics parameters need to be monitored, FBG sensor is an potential solution[4,5]. For cross-sensitivity problem between strain and temperature in FBG, A FBG signal demodulation system is designed to monitor the temperature and strain on the aircraft. FBG signal demodulation system mainly includes: light source, light demodulating equipment, processing circuit and demodulation program. To test performance of system, A FBG experiment was designed to verify the detecting ability of strain and temperature, the data is processed by Labview. 


\section{Principle of Demodulation and System Introduction}

FBG is a sensing device. When tested parameters are changed, its spectrum will also be changed. Since drift of center wavelength of reflection spectrum, the change of physical parameters can be calculated by detecting wavelength drift. When imputing FBG beam that meets the Bragg conditions will be reflected, reflection peak appears in the Bragg wavelength.

In the process of Strain measurement, wavelengths of Strain FBG and temperature compensation FBG (TC FBG) have a relationship with strain and temperature $[1,2,6]$.

$$
\begin{gathered}
\lambda_{T C}=\left(\alpha_{T C}+\zeta_{T C}\right) \cdot T \\
\lambda_{\varepsilon}=\left(1-P_{e}\right) \cdot \varepsilon+\left(\alpha_{\varepsilon}+\zeta_{\varepsilon}\right) \cdot T
\end{gathered}
$$

Where $\lambda_{T C}$ and $\lambda_{\varepsilon}$ are center wavelength of TC FBG and strain FBG respectively; $\alpha_{T C}$ and $\alpha_{\varepsilon}$ are thermal expansion coefficient of fiber, $\zeta_{T C}$ and $\zeta_{\varepsilon}$ are thermal optical coefficient of fiber, $P_{e}$ is photo elastic effect of material. $\varepsilon$ and $T$ are the strain and temperature of the environment.

After temperature compensation, change of strain FBG wavelength can be obtained by equation (1) and (2).

$$
\Delta \lambda=\lambda_{\varepsilon}-A \cdot \lambda_{T C}=\lambda_{\varepsilon}-\lambda_{T C} \cdot \frac{\alpha_{\varepsilon}+\zeta_{\varepsilon}}{\alpha_{T C}+\zeta_{T C}}=\left(1-P_{e}\right) \varepsilon
$$

When $\Delta \lambda$ is measured, strain $\varepsilon$ can be calculated.

\section{Demodulation System}

The FBG wavelength demodulation system scheme is based on the continuous dynamic scanning of tunable F-P filter. Using 3dB coupler, Bragg reflection light of FBG is coupled into the tunable fiber F-P filter (FPF), sawtooth voltage is loaded on FPF, in this way, FPF will scan the spectrum near the characteristic wavelength of grating. Therefore, the characteristics wavelength of the grating is the output wavelength of FPF scanning over zero. The demodulation system mainly consists of six parts: ASE broadband light source, tunable F-P filter, F-P etalon, coupler photoelectric detector. the data acquisition card and the data processing program on PC (Fig.1). ASE broadband light source and tunable F-P filter constitutes tunable laser. Its spectrum width is less than spectrum width of bragg reflection, tunable laser output wavelength is one-way change, its spectrum can be swept. The parameters of the device are showed in the table.1. 


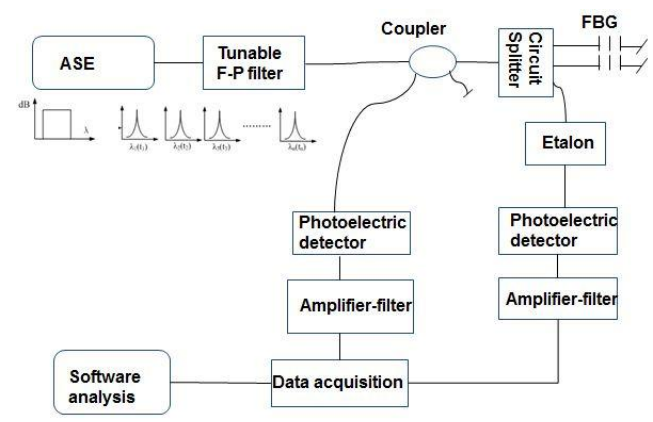

Fig.1 The demodulation system scheme

Tab. 1 The parameters of the device

\begin{tabular}{|c|c|c|c|c|}
\hline \multirow[b]{2}{*}{ ASE } & Wavelength range & \multicolumn{2}{|c|}{ Output power } & Spectral flatness \\
\hline & $1525 \sim 1565 \mathrm{~nm}$ & \multicolumn{2}{|c|}{$10 \mathrm{dBm}$} & $\leq 1.5 \mathrm{~dB}$ \\
\hline \multirow[b]{2}{*}{ FPF } & Wavelength range & FSR & Fineness & Input power \\
\hline & $1520 \sim 1570 \mathrm{~nm}$ & $145 \mathrm{~nm}$ & 4000 & $9 \mathrm{~mW}$ \\
\hline \multirow[b]{2}{*}{ Etalon } & Wavelength range & \multicolumn{2}{|c|}{ FSR } & Fineness \\
\hline & $1525 \sim 1565 \mathrm{~nm}$ & \multicolumn{2}{|c|}{$100 \mathrm{GHz}$} & 7 \\
\hline \multirow[b]{2}{*}{ DAQ Card } & Resolution & \multicolumn{2}{|c|}{ Accuracy } & Sampling frequency \\
\hline & 16bit & \multicolumn{2}{|c|}{$>0.015 \%$} & $500 \mathrm{KHz}$ \\
\hline \multirow[b]{2}{*}{ Photodetector } & operating voltage & \multicolumn{2}{|c|}{ dissipation power } & Operating temperature \\
\hline & $15 \mathrm{~V}$ & \multicolumn{2}{|c|}{$100 \mathrm{~mW}$} & $-40 \sim 70^{\circ} \mathrm{C}$ \\
\hline
\end{tabular}

We use Labview software to realize laser wavelength scanning, remotely control, data acquisition and signal analysis. Signal processing scheme is shown in Fig.2. In the data acquisition system, data collected is the form of voltage, so it must be restored to the corresponding physical quantities. Because of the influence of various interference and noise in internal and external system, noise and interfering signal may be added in the acquisition data, we can filter noise to ensure the precision of the acquisition system. Parameter configuration mainly includes sampling frequency $(300 \mathrm{kHz})$ and scanning time $(0.25 \mathrm{~s})$. Determining peak position refers to position from starting wavelength to etalon peak.

The system calls the module of peak detection to get position of sensing spectrum peak in discrete data sample points during a complete sawtooth scanning cycle. Combined with peak positioning search algorithm, spectral peak is precisely located. We apply amplitude weighting method to calculate peak of the sensing signal. We design program interface. Sensor is connected to system, reflection waveform is displayed on GUI as shown in Fig.3. 


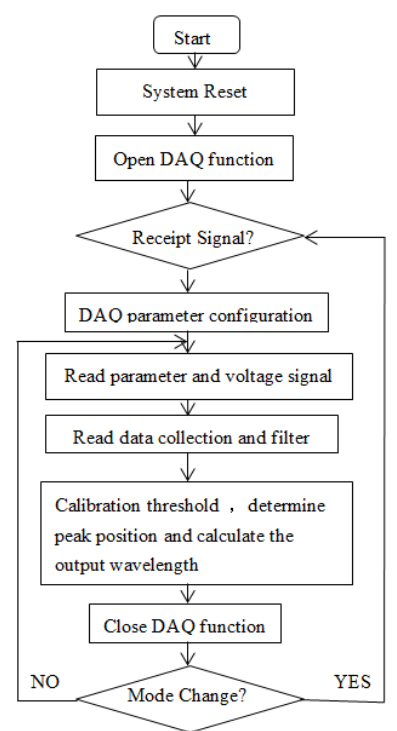

Fig.2 Signal processing scheme

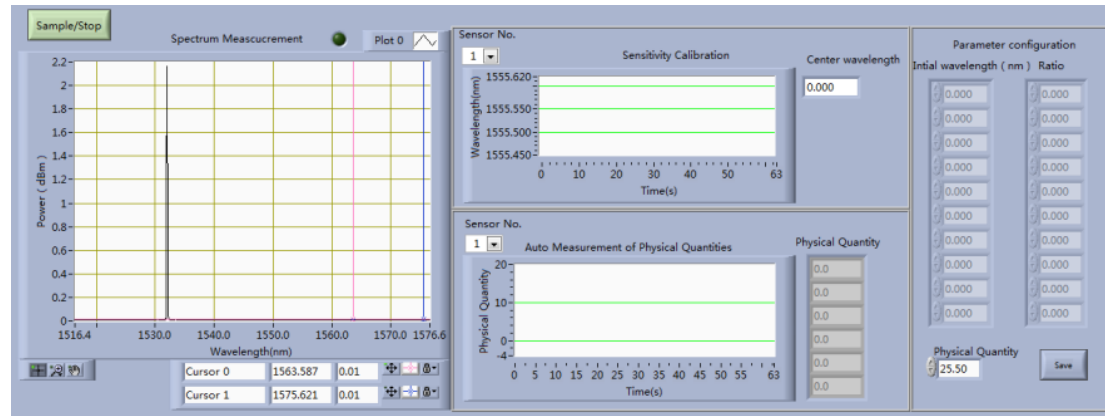

Fig.3 GUI and waveform display of the data

\section{Experimental Data Analysis}

We realize strain sensing with real-time temperature compensation using demodulation system. The strain FBG is pasted on centerline of cantilever beam to induct the strain of beam, and TC FBG is fixed on cantilever beam to realize temperature compensation. After FBGs are pasted, initial wavelength of strain FBG is $1541.650 \mathrm{~nm}$, initial wavelength of TC FBG is $1537.352 \mathrm{~nm}$. FBG Strain value is calculated by formula (3). The experiment is carried out at room temperature. Adopting spiral micrometer imposes stress on cantilever end, strain increment is $10 \mu \varepsilon$, strain is increased to $100 \mu \varepsilon$ gradually, we measure multiple times and record the change of the FBG wavelength with the temperature. Then 
strain FBG wavelengths $\left(\Delta \lambda_{\varepsilon}\right)$ are fitted with strain value (Fig.4 (a)). We observe that wavelengths of TC FBG don't drift, so it is not sensitive to strain. Goodness-of-fit of strain FBG is $99.922 \%$, strain can be sensed to strain FBG by the sensing structures. Wavelength moves towards long wavelength.

In order to realize strain sensing with real-time temperature compensation, we should determine temperature coefficient $(\alpha+\zeta)$ both FBGs. Sensors are placed in a constant temperature box. Thermostat method is used to measure the sensitive properties at different temperatures. Temperature increment is $5^{\circ} \mathrm{C}$, the temperature is increased to $70^{\circ} \mathrm{C}$, wavelengths $\left(\Delta \lambda_{T}\right)$ of strain $\mathrm{FBG}$ and TC FBG are fitted with temperature, so temperature coefficient of strain FBG is $10.76 \mathrm{pm} /{ }^{\circ} \mathrm{C}$, temperature coefficient of $\mathrm{TC}$ FBG is $10.96 \mathrm{pm} /{ }^{\circ} \mathrm{C}(\mathrm{Fig} .4$ (b)). Goodness-of-fits of strain FBG and TC FBG are $99.989 \%$ and $99.997 \%$ respectively. We also test that the system is well done between $-10^{\circ} \mathrm{C}$ and $0^{\circ} \mathrm{C}$.

When temperature increases from $25^{\circ} \mathrm{C}$ to $50^{\circ} \mathrm{C}$, strain is measured with real-time temperature compensation, $\lambda_{\varepsilon}-A \cdot \lambda_{T C}$ is fitted with strain, goodnessof-fit attains $99.99 \%$. Values of System measurement are basic coincidence with formula (3) calculation (Fig.4(c)). So TC FBG can provide accurate temperature compensation for strain FBG in strain measurement, and it can ensure a high precision measurement. In experiment, reflectance spectrum of FBG is shown in Fig.4 (d). The wavelength demodulation accuracy is 4pm (Fig.4 (e)). Curves Strain change is showed in Fig.4 (f).
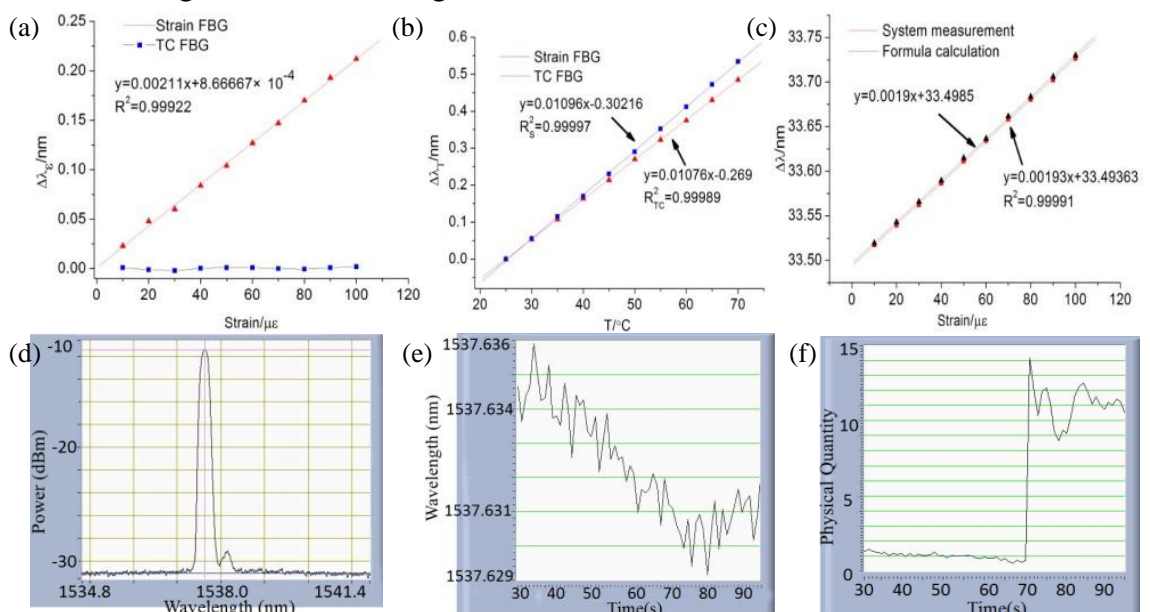

Fig.4 (a) Relations of both FBGs with the strain. (b) Relations of both FBGs with the temperature. (c) Relation between and the strain after temperature compensation. Where red solid line is strain FBG, blue solid line is TC FBG, black solid line is value of formula(3) calculation. (d) Reflectance spectrum of FBG. (e) The wavelength demodulation accuracy. (f) Strain change curves (applied stress ). 


\section{Conclusion}

For cross-sensitivity problem between strain and temperature in (FBG), we designed a FBG signal demodulation system and strain sensing with real-time temperature compensation can be completed to test performance of system. When temperature increases from $25^{\circ} \mathrm{C}$ to $50^{\circ} \mathrm{C}$, TC FBG can provide accurate temperature compensation for strain FBG in strain measurement. The system can be used as multi physical parameter monitoring device of the airplane status, and the sensing chip is low-cost. Not only have strain and temperature sensor of multi-point FBG be studied, more ideal results have been achieved.

\section{Acknowledgments}

The work was financially supported by the National Natural Science Foundation of China (NO. 61475094).

\section{References}

1. Jin Yong-xing, Liu Tao, Feng Tao, et al, Experimental study of temperature sensor systems for FBG based on Labview, Laser Journal, 30(1), pp.32-33, ( In Chinese, 2009).

2. Gui-ying Wang, Jie Jin, Cheng-gang Lv, A Novel Fiber Bragg Grating Sensors Interrogation System Based on Labview, Piezoelectrics \& Acoustooptics, vol34, pp.824-827, (In Chinese, 2012).

3. Yan-lin Xiong, Qiao-yi Li, Study on Wavelength Demodulation System with the Continuous Dynamic Scanning of Tunable DFB Laser, Internal Journal of Signal Processing, vol.7. pp.339-346, (In Chinese, 2014).

4. Liu Xue-dong, Xie xue-zheng, Xu Yuan-zhe, Study on fiber bragg grating sensor demodulation system based on Labview, Modern Instruments,2, pp.48-50, (In Chinese, 2010).

5 Guo T, Liu F, Liang X, et al.. Highly sensitive detection of urinary protein variations using tilted fiber grating sensors with plasmonic nanocoatings[J]. Biosensors and Bioelectronics, 78, pp.221-228, (In Chinese, 2016).

6. Luo Z, Wen $\mathrm{H}$, Guo $\mathrm{H}$, et al, A time-and wavelength-division multiplexing sensor network with ultra-weak fiber Bragg gratings[J], Optics Express, 21(19), 22799-22807, (In Chinese, 2013). 